\title{
Predictive value of isolated DLCO reduction in systemic sclerosis patients without cardio-pulmonary involvement at baseline
}

\author{
M. Colaci' ${ }^{1}$, D. Giuggioli' ${ }^{1}$, M. Sebastiani ${ }^{1}$, A. Manfredi', \\ F. Lumetti ${ }^{1}$, F. Luppi ${ }^{2}$, S. Cerri ${ }^{2}$, C. Ferri ${ }^{1}$ \\ ${ }^{1}$ Rheumatology Unit, University of Modena and Reggio Emilia, Medical School, \\ University Hospital of Modena, Italy; \\ ${ }^{2}$ Respiratory Disease Unit, University of Modena and Reggio Emilia, Medical School, \\ University Hospital of Modena, Italy
}

\begin{abstract}
SUMMARY
Impaired diffusing capacity of the lung for carbon monoxide (DLCO) was frequently observed in systemic sclerosis (SSc) patients, generally related to the presence of interstitial lung disease (ILD) and/or pulmonary arterial hypertension (PAH). However, in clinical practice abnormally low DLCO values may be found also in the absence of these SSc complications. The objective was to investigate the prospective clinical relevance of isolated DLCO reduction at baseline in SSc patients.

Ninety-seven SSc female patients (age at the diagnosis: $51.3 \pm 14.5$ years; disease duration: $10.4 \pm 6.6$ years; limited/diffuse skin subsets: 92/5), without any clinical, radiological (high resolution computed tomography), and echocardiographic manifestations of ILD or PAH at baseline, nor other lung or heart diseases able to affect DLCO, were recruited at our Rheumatology Centre.

Patients with DLCO <55\% (15 patients; group A) were compared with those with normal DLCO (82 patients; group B), at baseline and at the end of follow-up.

At baseline, patients of group A showed significantly higher percentage of anticentromere autoantibodies compared to group B $(13 / 15,86.6 \%$ vs $48 / 82,58.5 \%$; $\mathrm{p}=0.044)$. More interestingly, at the end of long-lasting clinical follow-up (11.6 \pm 6.7 years), pre-capillary PAH (right heart catheterization) solely developed in some patients of group A $(3 / 15,20 \%$ vs $0 / 82$; $\mathrm{p}=0.003)$.

In SSc patients, the presence at baseline of isolated, marked DLCO reduction $(<55 \%$ of predicted) and serum anticentromere autoantibodies might characterize a peculiar SSc subset that may precede the development of $\mathrm{PAH}$. Therefore, careful clinical follow-up of patients with isolated moderate-severe DLCO reduction should be mandatory.
\end{abstract}

Key words: Scleroderma; systemic sclerosis; DLCO; pulmonary arterial hypertension; lung fibrosis.

Reumatismo, 2015; 67 (4): 149-155

\section{INTRODUCTION}

ystemic sclerosis $(\mathrm{SSc})$ is a connective tissue disease characterized by excessive production of fibrotic tissue and endothelial dysfunction (1). The latter is responsible of widespread microvascular damage, leading to Raynaud's phenomenon, ischemic digital ulcers, pulmonary arterial hypertension (PAH), and/or renal crisis. Indeed, the clinical features of SSc patients may be widely variable, from subclinical vascular abnormalities, until severe life-threatening organ involvement (2). Therefore, long-term follow-up of $\mathrm{SSc}$ patients is recommendable, in order to diagnose $\mathrm{SSc}$ vascular complications precociously, with timely therapeutic approach. Namely, PAH is one of the most serious complications of SSc, characterized by very poor 5-year prognosis, generally worse than that observed in patients with idiopathic PAH (3). Besides, the availability of novel treatments for PAH, mainly the anti-endothelin antagonists, has given the hope to counteract this devastating complication; therefore, early diagnosis of SScassociated PAH may be decisive for the overall disease outcome (4). Transthoracic color-Doppler echocardiography is a noninvasive, reproducible, widely available tool to accurately estimate pulmonary arte- $\overline{\text { Corresponding author }}$

Michele Colaci

Rheumatology Unit,

University of Modena and Reggio Emilia

Policlinico di Modena

Via del Pozzo, 71 - 41100 Modena, Italy

E-mail: michelecolaci@virgilio.it 
rial pressure and to evaluate right ventricle performance (5). PAH is suspected if the peak velocity of tricuspid regurgitation is $>3$ meters/second, with consequent systolic pulmonary arterial pressure (PAPs) resulted $>35 \mathrm{mmHg}(6)$; in this case, the right heart catheterization is mandatory in order to confirm the vascular alteration and to distinguish between pre-capillary and post-capillary PAH $(5,6)$. Apart from cardiac ultrasonography, the presence of $\mathrm{PAH}$ can be suspected on the basis of the reduction of diffusing capacity of the lung for carbon monoxide (DLCO), mainly in absence of interstitial lung disease (ILD) (7). Recently, an international cross-sectional study (DETECT study) proposed an algorithm, in order to identify PAH in SSc patients and to minimise missed diagnoses (8). This tool includes forced vital capacity (FVC)\%/DLCO\% ratio, telangiectasias, anticentromere antibodies, NTproBNP, serum urate, and right axis deviation at electrocardiogram, as parameters to be considered in order to select patients who should perform echocardiography. In the second step, the referral to right heart catheterization was decided after considering right atrium area and tricuspid regurgitant jet velocity (TV) at echocardiography.

Interestingly, a few SSc patients may present an apparently isolated reduction of DLCO, without evidencing ILD at chest high-resolution computed tomography (HRCT) and with normal parameters (i.e., TV) at echocardiographic evaluation. Given the proper execution of the test, with correction for patients' hemoglobin levels (9), and by excluding other known causes that may reduce DLCO, the clinico-prognostic significance of this finding remains unknown. Hypothetically, SSc-related subclinical microvascular lung involvement could be suspected, which may potentially evolve into overt PAH. A predictive role of DLCO reduction, at different cut-off levels, for PAH development has been suggested with variable sensitivity and specificity (10); however, these previous studies analyzed the predictive value of DLCO reduction in the setting of patients with one or more cardio-pulmonary parameter altera- tions. On the other hand, the long-term outcome of SSc patients with isolated DLCO impairment was investigated by Steen et al. (11) in a cohort of 152 subjects recruited from 1972 to 1987 . Among these patients with a mean initial DLCO value of $48 \%$ of predicted, $11 \%$ developed PAH $(\mathrm{p}<0.01)$; moreover, the best predictor for isolated pulmonary hypertension was a $\mathrm{FVC} /$ DLCO ratio $>1.4$.

The aim of the present study is to investigate the clinico-prognostic relevance of isolated DLCO reduction during the follow-up of a cohort of SSc patients in absence of other cardio-pulmonary manifestations evaluated by means of chest HRCT and echocardiography.

\section{MATERIALS AND METHODS}

Ninety-seven SSc patients [mean age at the diagnosis $51.3 \pm 14.5$ standard deviation (SD) years, disease duration at the end of follow-up 10.4 $\pm 6.6 \mathrm{SD}$ years] referring at our University-based Rheumatology Centre from January $1^{\text {st }} 2002$ until December $31^{\text {th }} 2012$ were considered. All patients were diagnosed by trained rheumatologists with consolidated expertise in SSc; moreover, they fulfilled the 2013 American College of Rheumatology (ACR)/European League Against Rheumatism (EULAR) Classification Criteria for SSc (12). At baseline, the inclusion criteria to enter into the study were:

1) absence of dyspnea [New York Heart Association (NYHA) III-IV];

2) absence of ILD at chest HRCT;

3) FVC $>80 \%$;

4) normal echocardiography, namely without increase of the right atrial or ventricular areas and peak velocity of tricuspid regurgitation $<3$ meters/second;

5) absence of chronic obstructive pulmonary disease, heart failure or valvular heart diseases.

The patients were divided in two groups according to the DLCO values, considering as cut-off DLCO $<55 \%$, as proposed by Steen et al. (11).

Pulmonary function tests were performed using the spirometer MasterScreen Body 
(Jaeger-Toennies, Hoechberg, Germany); DLCO was measured by a single-breath technique and corrected for hemoglobin. Results were expressed as the percentage of predicted values; values $<80 \%$ of predicted were considered abnormal, using reference values of the Official Statement of the European Respiratory Society. Chest HRCT scans were obtained using the LightSpeed VCT (GE Healthcare, Buckinghamshire, UK). One-mm sections were acquired at $10-\mathrm{mm}$ intervals from apex to base of lungs, with the patients at full inspiration in a supine position. Additional scans in prone position were eventually recorded to evaluate the effect of gravity-dependent opacification. Echocardiographic measurements were performed with the subjects in partial left decubitus, by digital Vivid 7 ultrasound machine (GE-Vingmed, Horten, Norway).
A variable frequency phased-array transducer (2.5-3.5-4.0 $\mathrm{MHz}$ ) was used for two-dimensional, M-mode, and Doppler imaging. Left ventricular measures were obtained by M-mode with 2-D guidance at conventional levels, according to the American Society of Echocardiography recommendations. Right ventricle (RV) enddiastolic diameter was measured in apical four-chamber view at middle level. PAPs were estimated using Doppler recordings of tricuspid regurgitation, measuring the peak velocity of the tricuspid regurgitation jet. According to the data of the French Nationwide Prospective Multicenter Study, we considered significant the peak velocity that resulted $>3$ meters/second (6).

During the follow-up, spirometry with DLCO test and echocardiography were routinely performed yearly.

Table I - Clinical features of the whole systemic sclerosis series and 2 groups according to different DLCO values at baseline and at the end of follow-up.

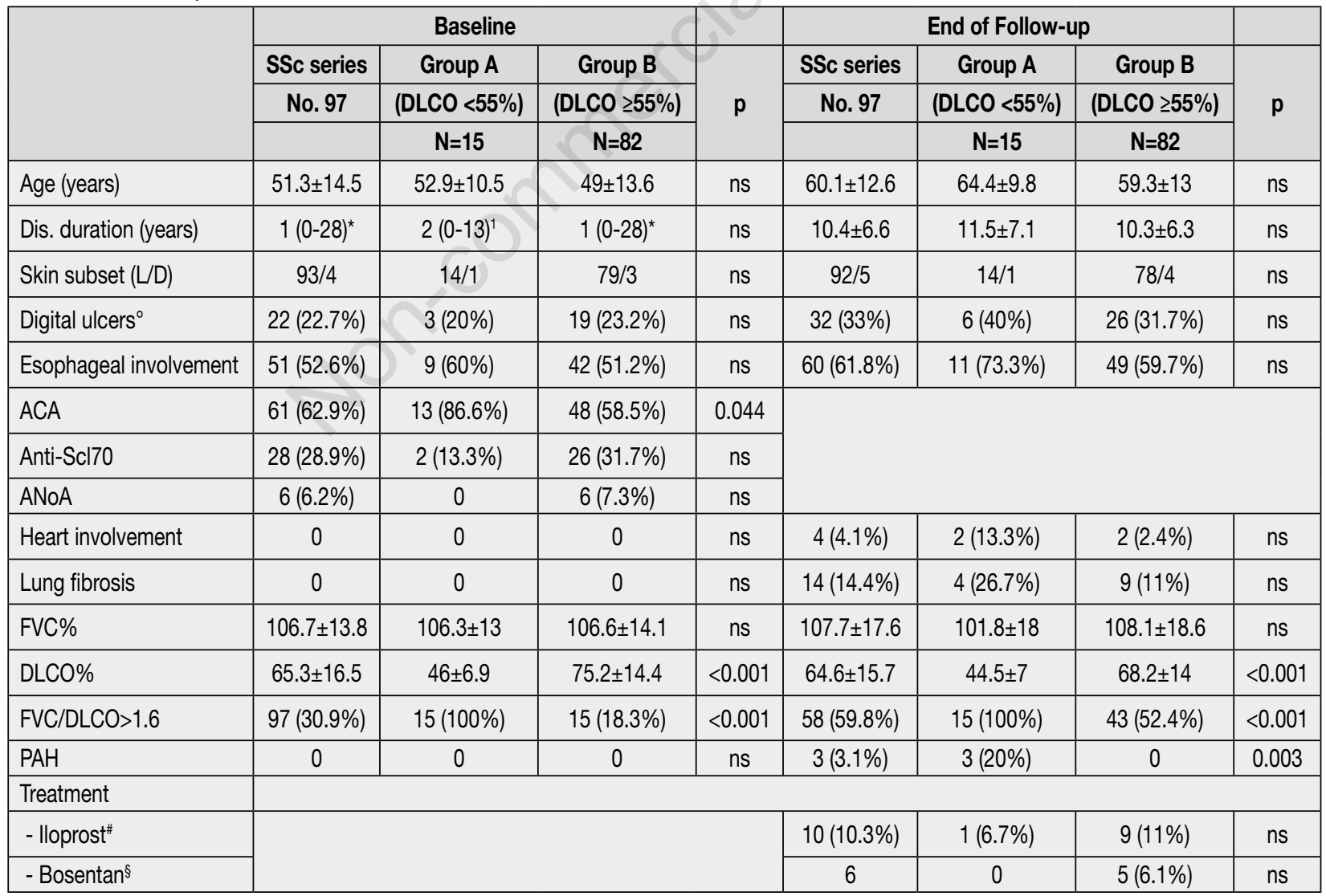

SSc, systemic sclerosis; DLCO, diffusing capacity of the lung for carbon monoxide; Dis., disease; FVC, forced vital capacity; PAH, pulmonary arterial hypertension. *Median (range); ' at least one episode during the time period preceding the patients' evaluation; \#for at least 5 years before the last patients' evaluation; §for at least 3 years before the last patients' evalutation. 
Statistical analysis was performed in order to investigate the possible correlations between SSc parameters and DLCO reduction. Values are given as mean \pm SD for normally distributed variables, or as median (range) for not normally distributed variables. Group values and proportions were compared by univariate analysis of variance (ANOVA) and Fisher's exact test, respectively.

\section{RESULTS}

Demographic and clinico-serological features of $97 \mathrm{SSc}$ patients included in the study are shown in Table 1 . Considering the 55\% cut-off for DLCO, 15 patients with these DLCO levels were included in group A, while 82 constituted group B (DLCO $\geq 55 \%$ ).

All patients were females; except from 5 cases, all individuals presented limited skin subset according to LeRoy's classification (13). Moreover, 61 had anticentromere, 28 anti-Scl70, and 7 anti-nucleolar autoantibodies. According to inclusion criteria, no subject showed cardio-pulmonary manifestations at baseline; consistently, mean FVC was $106.7 \pm 13.8 \%$. Evaluating possible differences between the 2 subsets, a significant higher frequency of ACA autoantibodies was found in the first group [13/15 (86.6\%) vs 48/82 (58.5\%); $\mathrm{p}=0.044]$.

At the end of the clinical follow-up (11.6 \pm 6.7 years), 14/97 patients developed ILD ( 4 in group A and 9 in group B; $p=n o t$ significant), represented by mild bi-basilar fibrosis with ground-glass pattern at chest $\mathrm{HRCT}$; indeed, mean $\mathrm{FVC} \%$ values did not decrease significantly.

Of note, three subjects in group A developed pre-capillary PAH $(\mathrm{p}=0.003)$, diagnosed by right heart catheterization. At the end of follow-up, another patient in group A presented a new increase of PAPs measured by means of cardiac ultrasonography, but suspected PAH was not confirmed at right heart catheterization. Interestingly, the disease duration of the patients with pre-capillary PAH was $>20$ years in 2 , and 8 years in the third one.

With regards to DLCO values, a significant deterioration (DLCO reduction $>10 \%$ of baseline values) was observed in 19 patients of group B during the follow-up; among them, 9 cases presented DLCO values $<55 \%$ at the end of the study. These subjects presented a disease duration of $14.1 \pm 8.1$ years; $7 / 9$ were ACA positive and 4 out of them presented digital ulcers; one patient developed a diffuse skin involvement and lung fibrosis; in the end, the last patient developed a scleroderma renal crisis.

Concordantly to DLCO subsetting, a FVC/ DLCO ratio $>1.6$ was invariably $(100 \%)$ observed in patients of group $\mathrm{A}$ and in $18.3 \%$ of group B, respectively $(\mathrm{p}<0.001)$; this statistically significant difference among two groups was also present at the end of follow-up (100\% vs $52.4 \%$, respectively; $\mathrm{p}<0.001)$.

The possible influence of vasoactive treatments was also analyzed, particularly some therapies (i.e., iloprost, bosentan) frequently used in SSc patients with PAH. Indeed, even because of the low number of patients treated, no statistical correlations were recorded.

\section{DISCUSSION}

In the present study, the prospective clinical significance of markedly low DLCO values, in absence of ILD or PAH at baseline, was investigated. In particular, SSc patients with isolated reduction of DLCO $(<55 \%)$ at the beginning of the follow-up showed higher prevalence of serum ACA autoantibodies compared to patients with normal DLCO values, as well as significantly increased incidence of PAH.

DLCO values represent a measure of the resistance encountered by gases during alveolar-capillary membrane diffusion. Therefore, every condition affecting the physiological gas exchanges may be estimated by means of DLCO test (14). Impaired DLCO was frequently reported in SSc patients; two main pathological conditions may be responsible of this alteration, namely ILD and PAH $(7,15)$. The first one may affect DLCO levels by reducing alveolar volume and/or by alveolar-capillary membrane thickening (16). This latter may be impaired also by $\mathrm{PAH}$, 
in which vascular remodelling leads to arterial stiffness and vessel wall tightening (17). In our study, we selected SSc patients without any evidence of ILD or PAH at baseline, in order to eliminate the main causes of DLCO impairment. However, a significant group of patients $(36 / 97 ; 37.1 \%)$ presented DLCO values $<70 \%$, not explained by other confounding factors, such as anaemia, smoke habit, or presence of other lung or heart diseases known to influence the test (14). Isolated DLCO reduction has been reported in SSc, especially in patients with limited skin subset (18), consistently with our findings.

In fact, DLCO impairment has been included among diagnostic parameters in patients with early $\operatorname{SSc}(8,19)$, also in absence of overt organ involvement. However, it is more difficult to attribute specific clinico-prognostic significance to this alteration, including possible early therapeutic attempts, in subjects generally asymptomatic or with very mild clinical symptoms.

On the other hand, the presence of moderate-severe reduction of DLCO, accordingly to the cut-off level here employed $(<55 \%)$, should induce at least patient's careful evaluation and monitoring. It was well established that DLCO reduction is one of the best predictor of PAH development, particularly in presence of $\mathrm{FVC}$ within normal range; namely, a FVC/ DLCO ratio of 1.6-1.8 helps to identify an increased likelihood of having PAH $(11,20)$. This issue has been focused by a multicenter cohort study for PAH assessment and recognition of outcomes (21); in particular, SSc patients with DLCO $<55 \%$ of predicted or FVC/DLCO $>1.6$, and PAPs $>40 \mathrm{mmHg}$, were considered at-risk for PAH (22). However, considering patients with complicating $\mathrm{PAH}$ versus those without, low DLCO alone at baseline was not able to distinguish between two groups, even if it remains an important predictor of this complication. Moreover, severely reduced DLCO $(<39 \%)$ at the time of PAH diagnosis seems to be a worse prognostic factor in
SSc patients. Consistently, Arunthari et al. (23) did not found significant correlations between right heart catheterization parameters and DLCO values in a cohort of 138 individuals, even when corrected for spirometric volumes.

Accordingly, we did not found a significant correlation between DLCO levels at baseline and PAH development considering a DLCO reduction $<70 \%$ as cut-off (data not shown); otherwise, focusing on the patients' subset with lower DLCO levels $(<55 \%)$ a statistically significant correlation was observed.

In the DETECT study (8), evaluating 466 SSc patients at increased risk of $\mathrm{PAH}, \mathrm{a}$ value of DLCO $<60 \%$ was an inclusion criterion; the $19 \%$ of these patients presented $\mathrm{PAH}$, consistently with the findings of our present series $(3 / 15,20 \%)$. Nonetheless, other parameters have been encompassed in the DETECT algorithm, since also echocardiography alone showed to be not so accurate for PAH identification (8). In this respect, considering our study, it cannot be excluded the possibility to have overlooked initial/mild PAH patients on the basis of normal findings obtained at the baseline echocardiography; however, this occurrence has been eventually offset by the long-lasting follow-up, including yearly echocardiographic evaluations.

With regards to other clinical SSc features, a trend to higher frequency of digital ulcers in patients with reduced DLCO was found at the end of follow-up. This findings, along with the significant presence of ACA autoantibodies and the pretension to PAH development, might define a peculiar SSc subset with significant vascular involvement. As regards skin subset, the low number of patients with diffuse skin involvement did not permit a conclusive statistical analysis.

In our patients' series, disease duration seems to be quite relevant in the development of PAH in two patients. In fact, $2 / 3$ subjects presented this complication after $>20$ years of follow-up; in particular, PAH appeared after 21 and 22 years from SSc diagnosis, respectively. This finding was not constantly reported in the few studies 
previously published; Hsu et al. (22) in the PHAROS study observed the development of PAH after 10.1 \pm 8.4 years from SSc onset. On the contrary, in a multicenter French study (24), PAH developed at any time after SSc diagnosis; in addition, patients with early-onset PAH were older at the time of SSc diagnosis compared to those without (25).

The possible usefulness of anti-endothelin receptor antagonists in preventing $\mathrm{PAH}$ remains still to be verified.

A limit of our study is the relatively low number of patients taken into account with respect to the low incidence of $\mathrm{PAH}$, one of the main SSc complication potentially correlated to reduced DLCO (26). However, this single Centre study firstly focused on particularly homogeneous subset of $15 \mathrm{SSc}$ patients, with isolated marked reduction of DLCO in the absence of ILD or PAH, thoroughly evaluated by means of wide clinical and instrumental assessment; in addition, the patients were compared to a large control group, at baseline and after a long-term clinical follow-up for the detection of late SSc complications.

Another limit of the study is that not all subjects underwent a right-heart-catheterization during the follow-up period, because of the lack of suspect of PAH at echocardiography; indeed, SSc patients who developed PAH could be at least underestimated, thus our findings might be further reinforced.

\section{CONCLUSIONS}

In conclusion, it could be hypothesized that the presence of clear-cut isolated DLCO impairment, serum ACA autoantibodies, and the propensity to develop PAH or digital ulcers might characterize a peculiar SSc subset; therefore, careful clinical follow-up of patients with isolated DLCO reduction $(<55 \%)$ should be mandatory in order to early detect the appearance of PAH, one of the most harmful SSc complication.

Conflict of interests: the authors declare to have no conflict of interests.

\section{REFERENCES}

1. Hudson M, Fritzler MJ. Diagnostic criteria of systemic sclerosis. J Autoimm. 2014; 48-49: 38-41.

2. Shah AA, Wigley FM. My approach to the treatment of scleroderma. Mayo Clin Proc. 2013; 88: 377-93.

3. Fisher MR, Mathai SC, Champion HC, et al. Clinical differences between idiopathic and scleroderma-related pulmonary hypertension. Arthritis Rheum. 2006; 54: 3043-50.

4. Hachulla E, Denton CP. Early intervention in pulmonary arterial hypertension associated with systemic sclerosis: an essential component of disease management. Eur Respir Rev. 2010; 19: 314-20.

5. Hoeper MM, Bogaard HJ, Condiffe R, et al. Definitions and diagnosis of pulmonary hypertension. J Am Coll Cardiol. 2013; 62: D42-50.

6. Hachulla E, Gressin V, Guillevin L, et al. Early detection of pulmonary arterial hypertension in systemic sclerosis. A French Nationwide Prospective Multicenter Study. Arthritis Rheum. 2005; 52: 3792-800.

7. Sivova N, Launay D, Wémeau-Stervinou L, et al. Relevance of partitioning DLCO to detect pulmonary hypertension in systemic sclerosis. PLoS One. 2013; 8: e78001.

8. Coghlan JG, Denton CP, Grünig E, et al. Evidence-based detection of pulmonary arterial hypertension in systemic sclerosis: the DETECT study. Ann Rheum Dis. 2014; 73: 1340-9.

9. Hughes JMB, Pride NB. Examination of the carbon monoxide diffusing capacity (DLco) in relation to its Kco and Va components. Am J Respir Crit Care Res. 2012; 186: 132-9.

10. Gladue H, Altorok N, Townsend W, et al. Screening and diagnostic modalities for connective tissue disease-associated pulmonary arterial hypertension: a systematic review. Semin Arthritis Rheum. 2014; 43: 536-41.

11. Steen VD, Graham G, Conte C, et al. Isolated diffusing capacity reduction in systemic sclerosis. Arthritis Rheum. 1992; 35: 765-70.

12. van den Hoogen F, Khanna D, Fransen J, et al. 2013 classification criteria for systemic sclerosis: an American College of Rheumatology/ European League Against Rheumatism collaborative initiative. Ann Rheum Dis. 2013; 72: 1747-55.

13. LeRoy EC, Black C, Fleischmajer R, et al. Scleroderma (systemic sclerosis): classification, subsets and pathogenesis. J Rheumatol. 1988; 15: 202-5.

14. Cotton DJ, Graham BL. Single-breath carbon monoxide diffusing capacity or transfer factor. In: Qutayba H, Joanne S, James M, eds. Physiologic basis of respiratory disease. Hamilton: BC Decker Inc. 2005; 659-9.

15. van der Lee I, Zanen P, Grutter JC, et al. Diffusing capacity for nitric oxide and carbon 
monoxide in patients with diffuse parenchymal lung disease and pulmonary arterial hypertension. Chest. 2006; 129: 378-83.

16. Hughes JM. The single breath transfer factor (TLCO) and the transfer coefficient (KCO): a window onto the pulmonary microcirculation. Clin Physiol Funct Imaging. 2003; 23: 63-71.

17. Chandra S, Shah SJ, Thenappan T, et al. Carbon monoxide diffusing capacity and mortality in pulmonary arterial hypertension. J Heart Lung Transplant. 2010; 29: 181-7.

18. Steen V, Medsger TA Jr. Predictors of isolated pulmonary hypertension in patients with systemic sclerosis and limited cutaneous involvement. Arthritis Rheum. 2003; 48: 516-22.

19. Trad S, Huong du LT, Frances C, et al. Impaired carbon monoxide diffusing capacity as a marker of limited systemic sclerosis. Eur J Int Med. 2011; 22: e80-6.

20. Denton CP, Calies JB, Phillips GD, et al. Comparison of doppler echocardiography and right heart catheterization to assess pulmonary hypertension in systemic sclerosis. Br J Rheumatol. 1997; 36: 239-43.

21. Hinchcliff M, Fischer A, Schiopu E, Steen V. Pulmonary hypertension assessment and recognition of outcomes in scleroderma (PHAROS): baseline characteristics and description of study population. J Rheumatol. 2011; 38: 2172-9.

22. Hsu VM, Chung L, Hummers LK, et al. Development of pulmonary hypertension in a high-risk population with systemic sclerosis in the Pulmonary Hypertension Assessment and Recognition of Outcomes in Scleroderma (PHAROS) cohort study. Semin Arthritis Rheum. 2014; 44: 55-62.

23. Arunthari V, Burger CD, Lee AS. Correlation of pulmonary function variables with hemodynamic measurements in patients with pulmonary arterial hypertension. Clin Respir J. 2011; 5: 35-43.

24. Hachulla E, Launay D, Mouthon L, et al. Is pulmonary arterial hypertension really a late complication of systemic sclerosis? Chest. 2009; 136: 1211-9.

25. Medsger TA Jr. Natural history of systemic sclerosis and the assessment of disease activity, severity, functional status, and psychologic well-being. Rheum Dis Clin North Am. 2003; 29: 255-73.

26. Iudici M, Codullo V, Giuggioli D, et al. Pulmonary hypertension in systemic sclerosis: prevalence, incidence and predictive factors in a large multicentric Italian cohort. Clin Exp Rheumatol. 2013; 31: 31-6. 